\title{
Knockdown of long non-coding RNA HOST2 inhibits the proliferation of triple negative breast cancer via regulation of the let-7b/CDK6 axis
}

\author{
YUEDONG ZHANG ${ }^{1}$, HONGWEI ZHANG ${ }^{1}$, HUIQING KANG ${ }^{2}$, WENJIE HUO ${ }^{1}$, \\ $\mathrm{YU} \mathrm{ZHOU}^{1}$ and YUCHAO ZHANG ${ }^{1}$ \\ Departments of ${ }^{1}$ Oncology and ${ }^{2}$ Pathology, Wuhai People's Hospital, Wuhai, \\ Inner Mongolia Autonomous Region 016000, P.R. China
}

Received May 2, 2018; Accepted September 6, 2018

DOI: $10.3892 / \mathrm{ijmm} .2018 .3995$

\begin{abstract}
The upregulation of long non-coding RNA (lncRNA) human ovarian cancer-specific transcript 2 (HOST2) has been identified in breast cancer. The present study aimed to investigate whether IncRNA HOST2 regulated the proliferation of triple negative breast cancer (TNBC) cells, and the underlying molecular mechanism. In total, 30 patients with primary TNBC, who were treated at Wuhai People's Hospital (Wuhai, China), were recruited for the present study. Reverse transcription-quantitative polymerase chain reaction analysis was used for the examination of gene expression levels. A Cell Counting kit-8 (CCK-8) assay was used for the detection of cell proliferation. Phases of the cell cycle were evaluated by flow cytometry. Western blot analysis was performed to detect protein expression levels. A dual luciferase activity assay was performed to examine the interaction between microRNA (miRNA) and the 3' untranslated region (UTR) of target mRNA. The results revealed increased expression levels of HOST2 in tumor tissues from patients with TNBC. A positive correlation was identified between the expression of HOST2 and cyclin-dependent kinase 6 (CDK6) in tumor tissues. The silencing of HOST2 induced cell proliferation inhibition and cell cycle redistribution in MDA-MB-231 and MDA-MB-468 TNBC cells. In these two cell lines, HOST2 silencing caused a decrease in the phosphorylation of RB1 and CDK6, which was observed at the mRNA and protein levels. However, the silencing of CDK6 did not alter the expression of HOST2. It was hypothesized and confirmed that let-7b, a previously reported target miRNA of HOST2, was able to directly bind to the 3 'UTR
\end{abstract}

Correspondence to: Dr Yuchao Zhang, Department of Oncology, Wuhai People's Hospital, 29 Huanghedong Street, Haibowan, Wuhai, Inner Mongolia Autonomous Region 016000, P.R. China

E-mail: zhangyuchaowhrh@yeah.net

Key words: IncRNA HOST2, triple negative breast cancer, let-7b/cyclin-dependent kinase 6 of CDK6 and repress its expression. The expression of let-7b was negatively correlated with the expression of HOST2 and CDK6 in tumor tissues. Overall, the data suggested that lncRNA HOST2 acts as an oncogene in TNBC via the upregulation of CDK6.

\section{Introduction}

Breast cancer is among the most common types of cancer in female patients, with a high global mortality rate (1). Over $1,000,000$ women are diagnosed each year, and $\geq 410,000$ diagnosed patients succumb to mortality, accounting for $\sim 14.0 \%$ of cases of cancer-associated mortality in women (2). In recent years, the incidence of breast cancer has increased by $3 \%$ each year in China, seriously threatening the health of women and causing a burden on society (3). According to gene expression profiling, breast cancer is categorized into four major subtypes: Luminal A, luminal B, human epidermal growth factor receptor 2 positive (Her2 ${ }^{+}$) and basal-like (4). Different subgroups are associated with different clinical outcomes (1). However, triple negative-breast cancer (TNBC), which is characterized by the lack of estrogen receptor, progesterone receptor, and expression of Her-2, is not effectively treated by endocrine therapy or Her-2-targeted therapy (5). TNBC has been a popular focus of investigation in clinical and scientific fields over the last decade (6). Since 2014, the correlation between the progression of TNBC and epigenetics has been investigated thoroughly (7). The identification of effective therapies for TNBC is urgent.

Long non-coding RNAs (lncRNAs), which are RNA molecules $>200 \mathrm{nt}$ in length, are important in a large number of cellular processes, including alterations of the cell- or tissue-specific expression profile, and the promotion of cell proliferation, metastasis and invasion (8). IncRNAs have been reported to be of significance in the diagnosis and treatment of multiple types of disease, specifically the tumorigenesis and progression of various types of cancer, including osteosarcoma and TNBC $(9,10)$. The functions of IncRNAs include modulation of gene methylation, activation of transcription, regulation of translation and other processes in conjugation with mRNAs and microRNAs (miRNAs) (11). Human ovarian 
cancer-specific transcript 2 (HOST2) is an lncRNA of 2.9 $\mathrm{kb}$, with no identified open reading frame (12). The lncRNA HOST2 is expressed at high levels in epithelial ovarian cancer, and the inhibition of HOST2 has been demonstrated to reduce the proliferation, migration and invasion of ovarian cancer cells (13).

Accordingly, the present study aimed to investigate the effects of lncRNA HOST2 on TNBC cell proliferation. This may be beneficial for understanding the developmental mechanisms of TNBC, and to identify potential biomarkers for the diagnosis and therapy of this disease.

\section{Materials and methods}

Cell lines and reagents. The MDA-MB-231 and MDA-MB-468 human TNBC cell lines, and the MCF10A normal human mammary epithelial cell line were purchased from American Type Culture Collection (Manassas, VA, USA). The MCF10A cells were maintained in DMEM/Ham's F-12 (Invitrogen; Thermo Fisher Scientific, Inc., Waltham, MA, USA) supplemented with $100 \mathrm{ng} / \mathrm{ml}$ cholera toxin (Sigma-Aldrich; Merck KGaA, Darmstadt, Germany), $20 \mathrm{ng} / \mathrm{ml}$ epidermal growth factor (Sigma-Aldrich, Merck KGaA), $0.01 \mathrm{mg} / \mathrm{ml}$ insulin (Sigma-Aldrich, Merck KGaA), $500 \mathrm{ng} / \mathrm{ml}$ hydrocortisone (Sigma-Aldrich, Merck KGaA), and 5\% chelex-treated horse serum (Sigma-Aldrich, Merck KGaA). The MDA-MB-468 and MDA-MB-231 cells were cultured in RPMI-1640 medium (Gibco; Thermo Fisher Scientific, Inc.) or L-15 (Gibco; Thermo Fisher Scientific, Inc.) The culture medium was supplemented with $10 \%$ (v/v) fetal bovine serum (FBS; Gibco; Thermo Fisher Scientific, Inc.), $100 \mathrm{U} / \mathrm{ml}$ streptomycin (Gibco; Thermo Fisher Scientific, Inc.), and $100 \mathrm{U} / \mathrm{ml}$ penicillin (Gibco; Thermo Fisher Scientific, Inc.) in an incubator with $5 \% \mathrm{CO}_{2}$ at $37^{\circ} \mathrm{C}$.

Tissue collection. In total, 30 patients with primary TNBC, treated at Wuhai People's Hospital, (Wuhai, China), participated in the present study. The primary TNBC tumor tissues and adjacent normal tissues were collected, washed, and frozen in liquid nitrogen immediately following surgery. No patients had received other treatments prior to surgery. Informed consent regarding the use of these samples was obtained from each patient. The present study was approved by the Ethics Committee of Wuhai People's Hospital. Patient information is summarized in Table I.

Cell transfection. A total of $2 \times 10^{5}$ cells/well were seeded in 6-well plates and incubated in an incubator at $37^{\circ} \mathrm{C}$ with $5 \% \mathrm{CO}_{2}$ for $24 \mathrm{~h}$ to a confluence of $50-60 \%$. The cells were transfected with control small interfering (si)RNA and HOST2 siRNA (Invitrogen; Thermo Fisher Scientific, Inc.) using Lipofectamine RNAiMAX (Invitrogen; Thermo Fisher Scientific, Inc.) according to the manufacturer's protocol, and incubated at room temperature for $20 \mathrm{~min}$. The $1 \mathrm{nc}-\mathrm{HOST} 2$ siRNA sequences used were as follows: HOST2 siRNA, 5'-GACTAAACAAGGTCTTAATTT-3' and HOST2 siRNA\#, 5'-TGACTAAACAAGGTCTTAATTT-3'. The negative control siRNA sequence used was 5'-TTCTCCGAACGTGTC ACGTTT-3'. The transfected cells were washed with PBS three times, followed by incubation with $1.5 \mathrm{ml}$ fresh serum/antibiotic-free MEM for $48 \mathrm{~h}$ for the subsequent experiments.
Table I. Association between the expression of HOST2 and patient clinical and pathological characteristics.

\begin{tabular}{|c|c|c|c|c|}
\hline \multirow{2}{*}{$\begin{array}{l}\text { Clinicopathological } \\
\text { feature }\end{array}$} & \multirow{2}{*}{$\begin{array}{l}\text { Cases } \\
\text { (n) }\end{array}$} & \multicolumn{2}{|c|}{$\begin{array}{l}\text { Expression } \\
\text { of HOST2 }\end{array}$} & \multirow[b]{2}{*}{ P-value } \\
\hline & & Low (n) & High (n) & \\
\hline Age (years) & & & & 0.699 \\
\hline$\leq 45$ & 10 & 4 & 6 & \\
\hline$>45$ & 20 & 11 & 9 & \\
\hline Tumor size $(\mathrm{cm})$ & & & & 0.710 \\
\hline$\leq 2$ & 12 & 7 & 5 & \\
\hline$>2$ & 18 & 8 & 10 & \\
\hline TNM stage & & & & $0.023^{\mathrm{a}}$ \\
\hline I & 8 & 7 & 1 & \\
\hline II & 10 & 5 & 5 & \\
\hline III & 12 & 3 & 9 & \\
\hline $\mathrm{Ki}-67$ & & & & 0.139 \\
\hline$\leq 20$ & 17 & 11 & 6 & \\
\hline$>20$ & 13 & 4 & 9 & \\
\hline
\end{tabular}

${ }^{a} \mathrm{P}<0.05$ was considered to indicate a statistically significant difference. HOST2, human ovarian cancer-specific transcript 2; TNM, tumor-node-metastasis.

Western blot analysis. Proteins were extracted from the cell lysates using RIPA buffer (Roche Diagnostics, Shanghai, China). The protein concentration was determined using a BCA kit (Beyotime Institute of Biotechnology, Shanghai, China). A total of $10 \mu \mathrm{g}$ protein per sample was separated by $10 \%$ polyacrylamide gel electrophoresis Subsequently, the protein was transferred onto polyvinylidene fluoride (PVDF) membranes (EMD Millipore). The PVDF membranes were blocked with 5\% bovine serum albumin (Sigma-Aldrich, Merck KGaA) at room temperature for $1 \mathrm{~h}$, and incubated with primary antibodies against CDK6 (cat. no. 13331; 1:1,000) and GAPDH (cat. no. 5174; 1:5,000) (Cell Signaling Technologies, Inc., Danvers, MA, USA) at $4^{\circ} \mathrm{C}$ overnight. The following day, the PVDF membranes were washed three times with TBS-Tween, incubated with a secondary antibody (horseradish peroxidase-linked anti-rabbit IgG; Cell Signaling Technologies, Inc., cat. no. 7074, 1:10,000) at room temperature for $1 \mathrm{~h}$, and washed three times again with TBST. Finally, the proteins were visualized using chemiluminescence reagent and images were captured with the Bio-Rad Gel Dol EZ system (Bio-Rad Laboratories, Inc., Hercules, CA, USA).

Reverse transcription-quantitative polymerase chain reaction $(R T-q P C R)$ analysis. Total RNA was extracted from the tissues or cells with TRIzol (Invitrogen; Thermo Fisher Scientific, Inc.), and cDNA was synthesized using a reverse transcription kit (Promega Corporation, Madison, WI, USA).

To evaluate the expression of let- $7 \mathrm{~b}$, the PCR reaction contained $5 \mu \mathrm{l}$ 2X SYBR-Green Taq reaction liquid, $0.2 \mu \mathrm{l}$ Rox II, $0.2 \mu \mathrm{l}$ sense primer, $0.2 \mu \mathrm{l}$ antisense primer, $1 \mu \mathrm{l} \mathrm{cDNA}$ and $3.4 \mu \mathrm{ldd} \mathrm{d}_{2} \mathrm{O}$. The thermocycling conditions were as follows: 

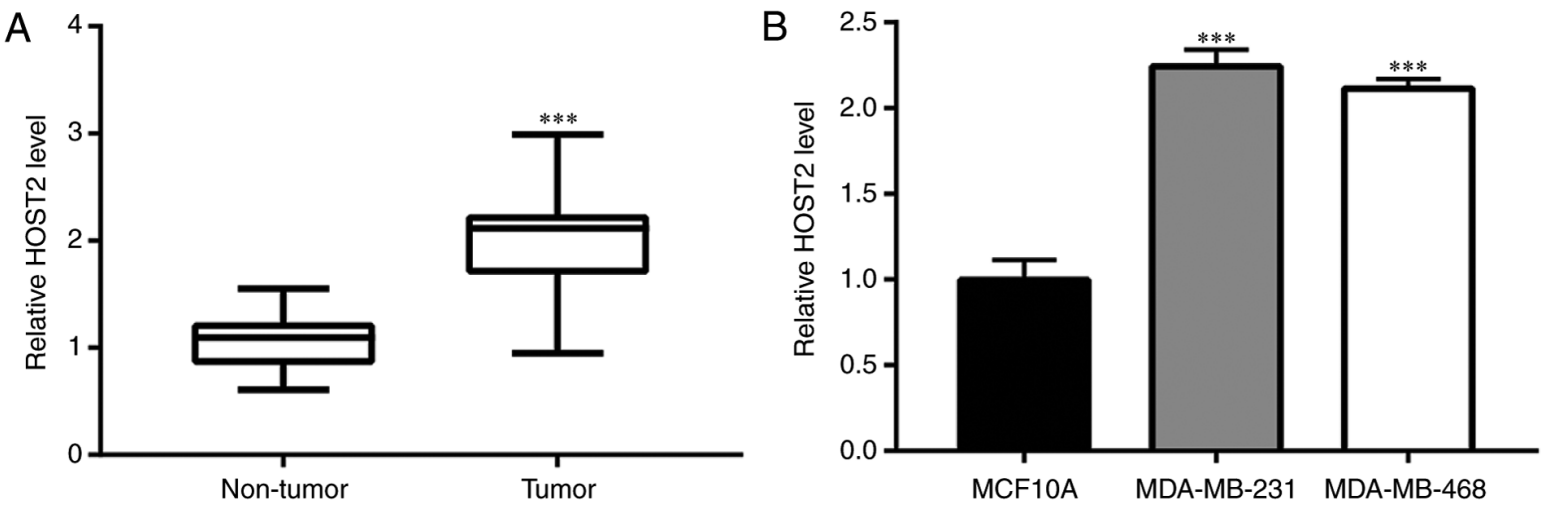

Figure 1. Overexpression of lncRNA HOST2 in TNBC tumor tissues and cell lines. (A) Compared with matched normal tissues, HOST2 levels were higher in tumor tissues from patients with TNBC. (B) Compared with the MCF-10A immoral epithelial breast cell line, expression of HOST2 was higher in MDA-MB-231 and MDA-MB-468 TNBC cell lines. ${ }^{* * *} \mathrm{P}<0.0001$. TNBC, triple negative breast cancer; HOST, human ovarian cancer-specific transcript 2.

$94^{\circ} \mathrm{C}$ for $10 \mathrm{sec}, 94^{\circ} \mathrm{C}$ for $5 \mathrm{sec}$, and $60^{\circ} \mathrm{C}$ for $34 \mathrm{sec}(40$ cycles). The primers were designed using Primer 5.0 software (Premier Biosoft International, Palo Alto, CA, USA) according to the gene sequences in the Genebank database (www.ncbi.nlm.nih.gov/), the sequences were as follows: let-7b, forward 5'-TGAGGTAGT AGGTTGTGTGGTT-3' and miR universal reverse 5'-GTG CAGGGTCCGAGGT-3'; U6, forward 5'-CTCGCTTCGGCA GCACA-3' and reverse 5'-AACGCTTCACGAATTTGCGT-3'. U6 was used as an internal control.

For the evaluation of HOST2 and CDK6, the PCR reaction consisted of $5 \mu \mathrm{l} 2 \mathrm{X}$ super Real, $0.3 \mu \mathrm{l}$ forward primer, $0.3 \mu \mathrm{l}$ reverse primer, $2 \mu \mathrm{l}$ reverse transcription products, $1 \mu \mathrm{l}$ 10X ROX and $1.4 \mu \mathrm{l}$ water without RNase. The thermocycling conditions were as follows: $95^{\circ} \mathrm{C}$ for $15 \mathrm{~min}$, followed by $95^{\circ} \mathrm{C}$ for $10 \mathrm{sec}$, annealing for $30 \mathrm{sec}$ and extension for $30 \mathrm{sec}$ (40 cycles). The primers were designed by Primer 5.0 software, according to the gene sequences in the Genebank database, and the sequences are as follows: HOST2 forward, 5'-CTCAAA TCAATCACGACCCT-3' and reverse, 5'-AATGTAGCAGGA CGAGCC-3'; CDK6 forward, 5'-TGGAGACCTTCGAGC ACC-3' and reverse 5'-CACTCCAGGCTCTGGAACTT-3'; and GAPDH forward, 5'-GGTGAAGGTCGGAGTCA ACG-3' and reverse, 5'-CAAAGTTGTCATGGATGHACC-3'. GAPDH was used as an internal control. The gene expression levels were quantified using the $2^{-\Delta \Delta \mathrm{Cq}}$ method (14).

Flow cytometry with PI staining. At 48 h post-transfection, the cells were washed three times with ice-cold PBS and centrifuged at $4^{\circ} \mathrm{C}$ for $10 \mathrm{mins}(1,000 \mathrm{x} \mathrm{g})$. The cells were re-suspended and adjusted to a density of $1 \times 10^{5}$ cells $/ \mathrm{ml}$. The cells were fixed with $1 \mathrm{ml}$ ice-cold $75 \%$ ethanol overnight at $4^{\circ} \mathrm{C}$. The following day, the cells were washed three times with PBS, followed by aspiration of the supernatant. The cells were then treated with $100 \mu \mathrm{l}$ RNaseA in a water bath at $37^{\circ} \mathrm{C}$ for $30 \mathrm{~min}$ in the dark, followed by staining with $400 \mu \mathrm{l}$ PI and incubation at $4^{\circ} \mathrm{C}$ for $30 \mathrm{~min}$ in the dark. The phases of the cell cycle were evaluated by flow cytometry (Coulter Corporation Co., Ltd., California, USA) using red fluorescence at $488 \mathrm{~nm}$.

Cell Counting kit-8 (CCK-8) assay. When the transfected cells reached $60 \%$ confluence, they were washed by PBS three times and digested into a single-cell suspension using $0.25 \%$ trypsin.
The cells were then seeded in a 96-well plate $(200 \mu \mathrm{l} /$ well $)$ at a density of $6 \times 10^{3}$ cells/well, and cultured for $48 \mathrm{~h}$ at $37^{\circ} \mathrm{C}$. Following this, $10 \mu \mathrm{l}$ CCK-8 solution was added to each well, and the cells were cultured for another $2 \mathrm{~h}$ at $37^{\circ} \mathrm{C}$. The optical density (OD) in each well was evaluated at a wavelength of $450 \mathrm{~nm}$.

Overexpression of let-7b. miRNA (miR)-NC mimics and let-7b mimics were synthesized by Guangzhou Ribobio Co., Ltd. (Guangzhou, China). To induce let-7b overexpression, the let-7b mimics were mixed with Lipofectamine 2000 (Invitrogen; Thermo Fisher Scientific, Inc.) in serum-free medium for 15 mins, and then added to the cells.

Bioinformatics analysis and dual luciferase activity assay. The bioinformatics analysis of the potential binding site between let-7b and CDK6 3'UTR was performed on miRDB (http://mirdb.org/). Oligonucleotides containing the CDK6 cDNA fragment, including let-7b binding sites, were amplified from the MDA-MB-231 cells and cloned into pmirGLO plasmids (Promega Corporation). Mutant CDK6 (pmirGLO-CDK6-MUT) served as a negative control and was generated by site-directed mutagenesis PCR using platinum pfx DNA polymerase, according to the manufacturer's protocol. The luciferase reporter plasmid and let-7b mimics or miR-NC mimics were co-transfected into MDA-MB-231 cells using Lipofectamine 2000. At $48 \mathrm{~h}$ post-transfection, the relative luciferase activity was examined using a Dual-Luciferase Reporter Assay system (Promega Corporation) in a luminometer. For the analysis of let-7b and HOST2 binding, 1,500-2,000 nt of the HOST2 sequence was amplified from MCF10A cDNA and annealed into the pGL3 plasmid (Promega Corporation). Mutations were introduced via site-directed mutagenesis PCR using platinum pfx DNA polymerase to construct pGL3-HOST2 mutant (Mut). In the dual luciferase reporter assay, the MAD-MB-231 and MDA-MB-468 cells were co-transfected with pGL3-HOST2 wild-type (WT) or pGL3-HOST2 Mut in combination with let-7b mimics or miR-NC mimics using Lipofectamine 2000. After $48 \mathrm{~h}$, the relative luciferase activity was examined using a Dual-Luciferase Reporter Assay system (Promega Corporation) in a luminometer. 

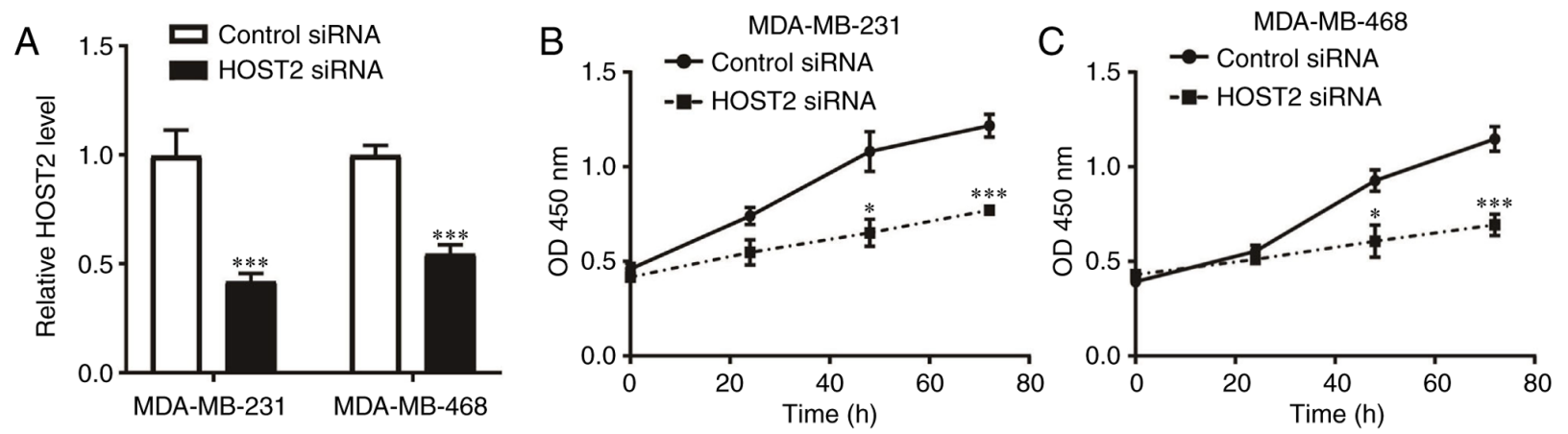

$\mathrm{D}$

MDA-MB-231
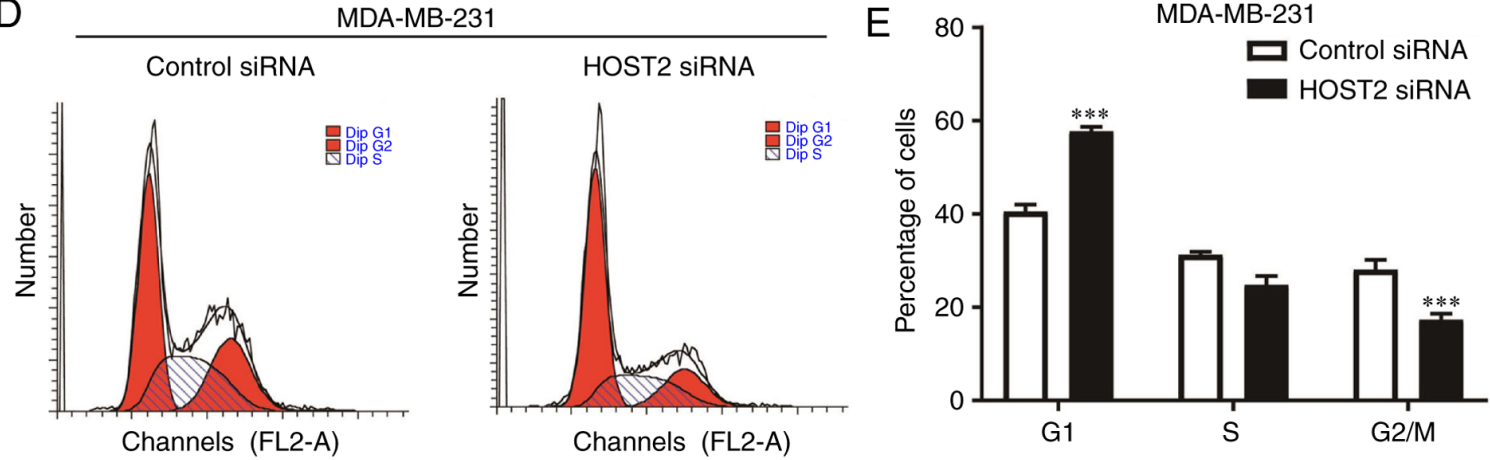

$\mathrm{F}$

MDA-MB-468
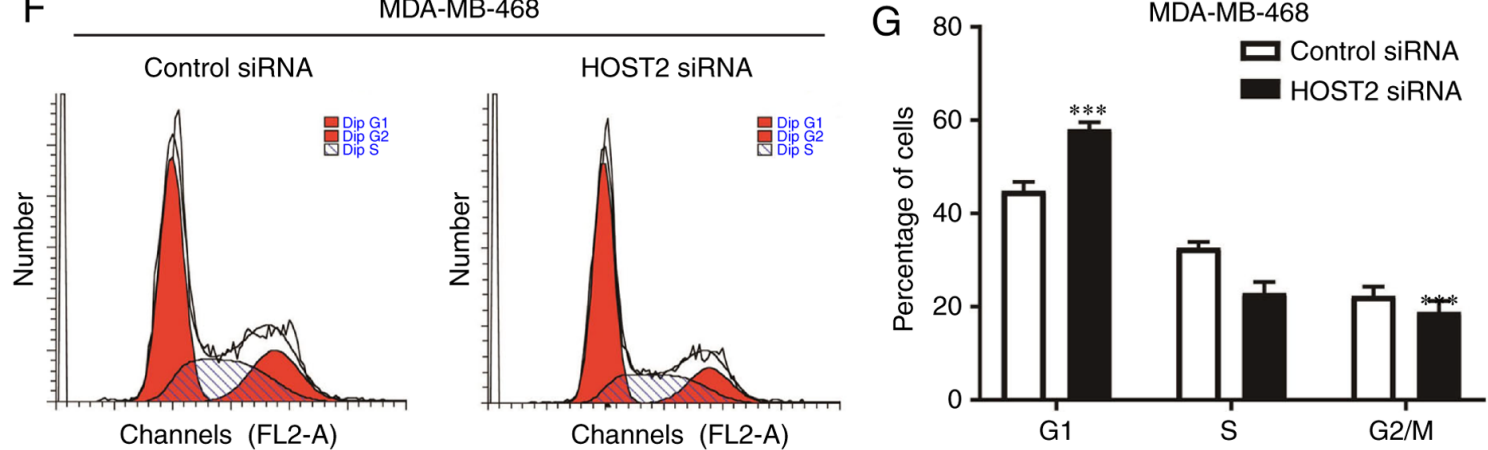

Figure 2. Silencing of HOST2 induces cell growth arrest and redistribution of cell cycle in triple negative breast cancer cells. (A) Transfection of HOST2 siRNA silenced the expression of HOST2 in MDA-MB-231 and MDA-MB-468 cells. Silencing of HOST2 inhibited cell proliferation of (B) MDA-MB-231 and (C) MDA-MB-468 cells. (D) Flow cytometry revealed (E) HOST2 knockdown increased cells enriched in the $\mathrm{G}_{1}$ phase and decreased cells accumulated in the $\mathrm{G}_{2} / \mathrm{M}$ phase in MDA-MB-231 cells. (F) Flow cytometry revealed (G) HOST2 knockdown increased cells enriched in the $\mathrm{G}_{1}$ phase in MDA-MB-468 cells.

${ }^{* * * *} \mathrm{P}<0.0001$. HOST, human ovarian cancer-specific transcript 2; siRNA, small interfering RNA.

Statistical analysis. SPSS 21.0 (IBM SPSS, Armonk, NY, USA) was used for statistical analysis. The data are presented as the mean \pm standard deviation. Comparisons of two groups were made using Student's t-test. One-way analysis of variance followed by NewmanKeuls post hoc analysis was used to compare the means of multiple groups. The association between HOST2 levels and patient clinical and pathological characteristics were analysed using Chi-square test. $\mathrm{P}<0.05$ was considered to indicate a statistically significant difference. The experiments were repeated three times.

\section{Results}

Overexpression of HOST2 in TNBC tumor tissues and cell lines. To evaluate the role of HOST2 in TNBC, RT-qPCR analysis was performed to compare the expression of HOST2 between TNBC tumor tissues and adjacent normal tissues. Compared with the adjacent normal tissues, the expres- sion of HOST2 was significantly increased in the TNBC tumor tissues (Fig. 1A). The expression of HOST2 was also detected in MCF10A normal breast epithelial cells and in the MDA-MB-231 and MDA-MB-468 TNBC cell lines. Increased expression of HOST2 was observed in the TNBC cells, compared with the MCF10A cells (Fig. 1B). The potential clinicopathological implications of the expression of HOST2 were also investigated in 30 patients with TNBC. The patients were divided into low and high HOST2 expression groups. Analysis of the association between the expression of HOST2 and patient clinicopathological parameters revealed that a high expression of HOST2 was closely associated with TNBC tumor-node-metastasis stage. However, the expression of HOST2 was not associated with age, tumor size or the expression of Ki-67 (Table I).

Overall, the above results suggested the oncogenic potential of HOST2, and that it is involved in the progression of TNBC. 

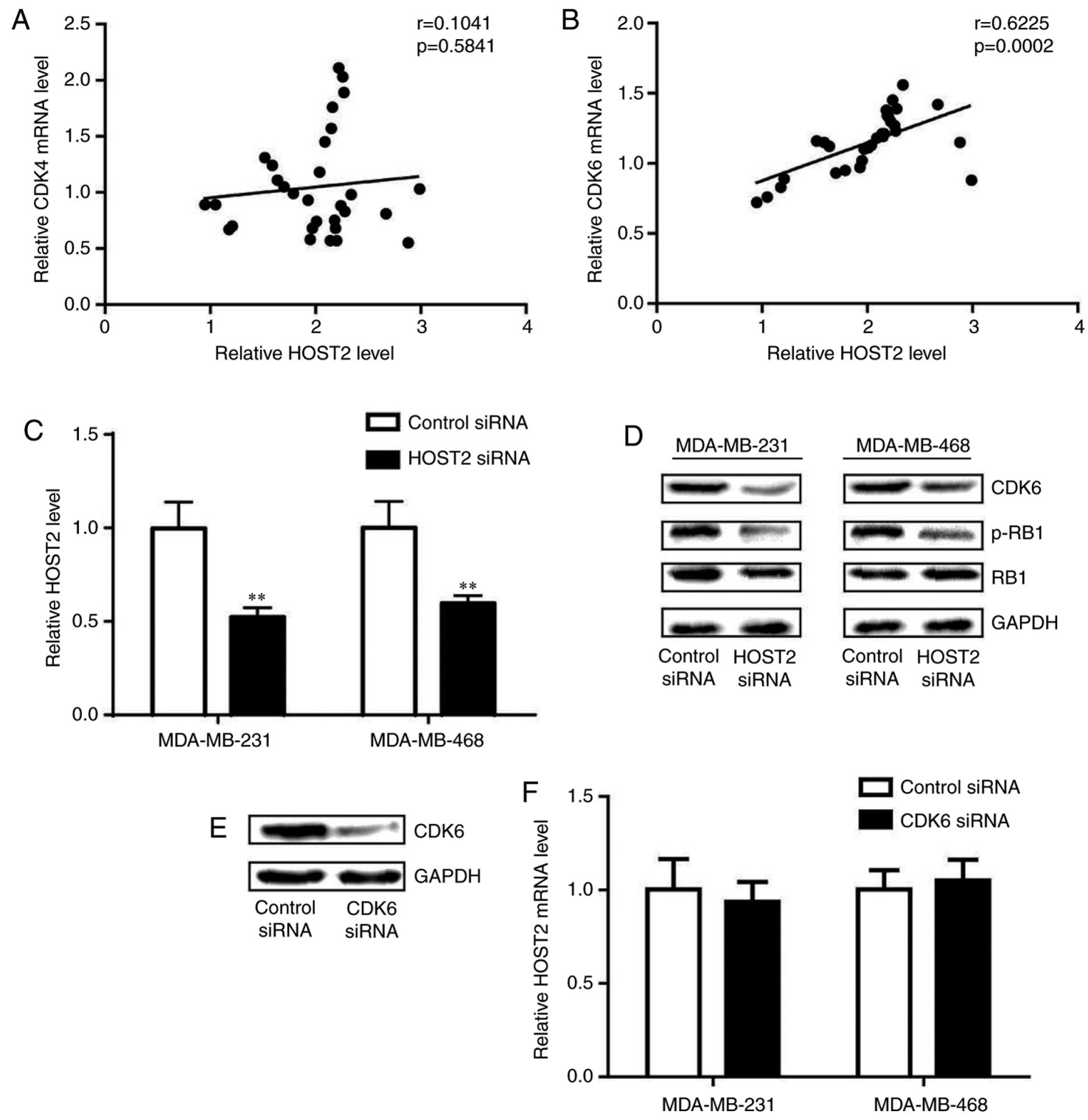

Figure 3. HOST2 positively regulates the expression of CDK6 in TNBC cells. Analysis of key regulators (A) CDK4 and (B) CDK6 of $\mathrm{G}_{1}$ phase expression in TNBC tumor tissues indicated that levels of CDK6, but not CDK4, were positively associated with HOST2 levels in tumor tissues. (C) HOST2 silencing led to decreased of mRNA levels of CDK6 in MDA-MB-231 and MDA-MB-468 cells. (D) HOST2 silencing decreased the protein level of CDK6 and dephosphorylated RB1 in MDA-MB-231 and MDA-MB-468 cells. (E) Transfection with CDK6 siRNA decreased levels of CDK6. (F) Silencing of CDK6 did not alter the expression of HOST2 in the cell lines. ${ }^{* *} \mathrm{P}<0.01$. HOST, human ovarian cancer-specific transcript 2; siRNA, small interfering RNA; CDK, cyclin-dependent kinase; p-, phosphorylated.

Silencing of HOST2 inhibits cell proliferation and cell cycle arrest in TNBC cells. The silencing of HOST2 was induced to assess the function of HOST2 in TNBC cells. HOST2 siRNA transfection decreased the expression of HOST2 in the MDA-MB-231 and MDA-MB-468 cells (Fig. 2A). The CCK-8 assay indicated that HOST2 silencing led to a significant decrease in the proliferative rate of the MDA-MB-231 and MDA-MB-468 cells (Fig. 2B and C). Cell cycle arrest was a major reason for cell proliferation inhibition. Using flow cytometric analysis, it was found that HOST2 silencing increased the proportion of MDA-MB-231 and MDA-MB-468 cells in the G1 phase (Fig. 2D-G), but did not affect the apoptotic cell rate of the MDA-MB-231 and MDA-MB-468 cells (data not shown). Therefore, HOST2 may promote TNBC progression through regulation of the G1-S checkpoint.
Silencing of HOST2 represses the expression of CDK6 in TNBC cells. The cyclin D-CDK4/6 complex has been demonstrated to be pivotal in controlling the G1-S checkpoint (15). The analysis of gene expression levels in TNBC tumor tissues revealed that the expression of CDK6 was positively correlated with that of HOST2, whereas the expression of CDK4 was not (Fig. 3A and B). Consistently, silencing of the expression of HOST2 decreased the expression of CDK6 in the MDA-MB-231 and MDA-MB-468 cells (Fig. 3C). During the G1-S phase, the phosphorylation of RB1 by CDK6 permits the transcription of $\mathrm{S}$ phase genes, and is required for DNA replication (16). Western blot analysis revealed that the protein expression level of CDK6 and the phosphorylation of RB1 were reduced following HOST2 silencing in the MDA-MB-231 and MDA-MB-468 cells (Fig. 3D). To determine whether CDK6 regulated HOST2, CDK6 siRNA was transfected into TNBC cells. The trans- 

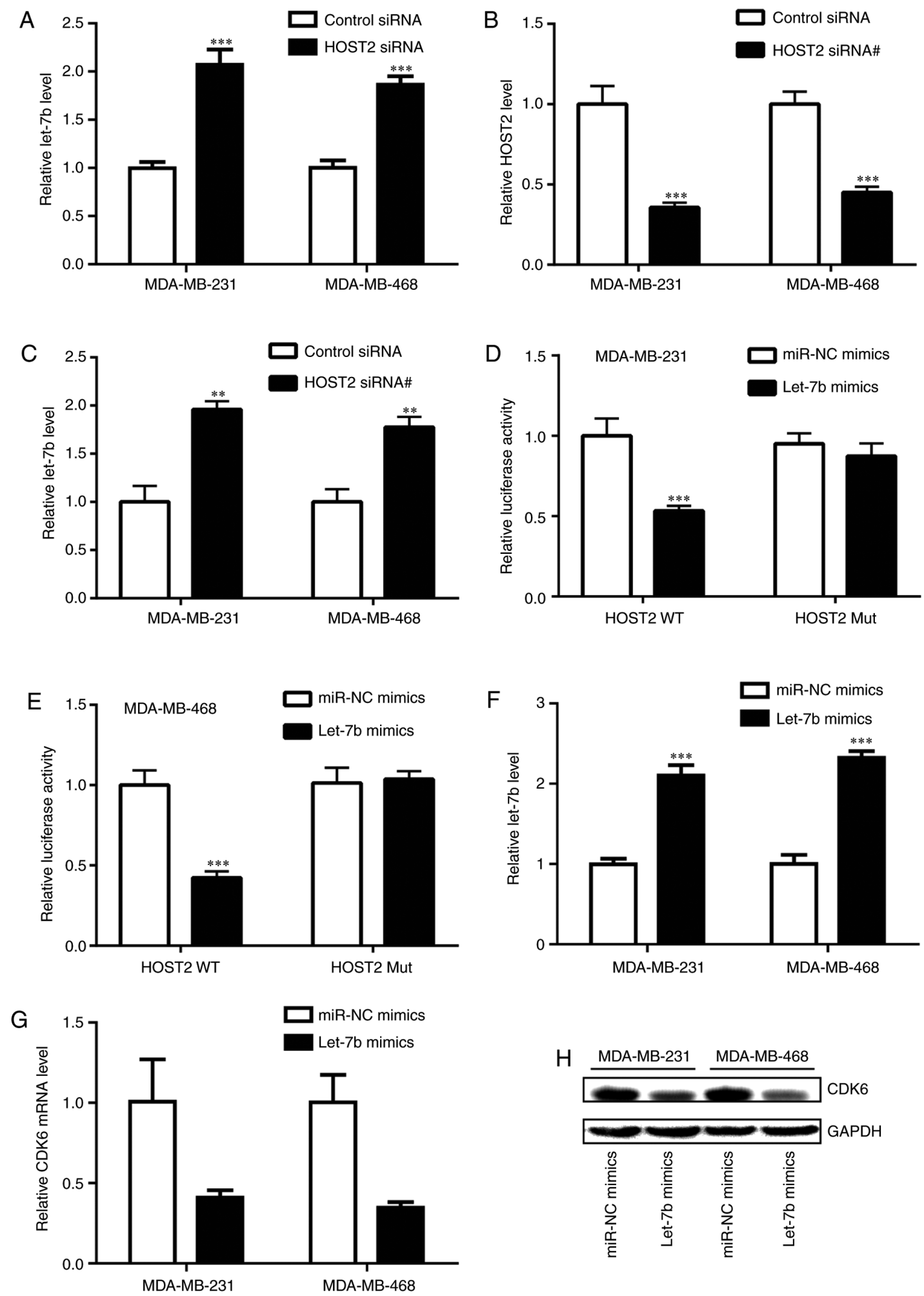

Figure 4. HOST2 represses the expression of let-7b to regulate CDK6 levels. (A) Silencing of HOST2 increased the expression of let-7b in MDA-MB-231 and MDA-MB-468 cells. (B) Transfection with HOST2 siRNA\# reduced the expression of HOST2 in MDA-MB-231 and MDA-MB-468 cells. (C) Expression of let-7b was elevated upon transfection with HOST2 siRNA\# in MDA-MB-231 and MDA-MB-468 cells. Transfection of let-7b mimics reduced the relative luciferase activity of pGL3-HOST2 WT in (D) MDA-MB-231 and (E) MDA-MB-468 cells. (F) Transfection with let-7b mimics elevated the level of let-7b in MDA-MB-231 and MDA-MB-468. Overexpression of let-7b decreased CDK6 at the (G) mRNA and (H) protein levels in MDA-MB-231 and MDA-MB-468. ${ }^{* *} \mathrm{P}<0.01 ;{ }^{* * *} \mathrm{P}<0.0001$. HOST, human ovarian cancer-specific transcript 2; siRNA, small interfering RNA; CDK, cyclin-dependent kinase; miR, microRNA; $\mathrm{NC}$, negative control.

fection of CDK6 siRNA decreased the expression of CDK6 (Fig. 3E). CDK6 silencing did not alter the level of HOST2 in the MDA-MB-231 or MDA-MB-468 cells (Fig. 3F).
Collectively, the above data suggested that CDK6, a key regulatory kinase in the cell cycle, was negatively regulated by HOST2 in TNBC cells. 
A

$\begin{aligned} \text { CDK6 3'UTR } & \text { 5' UAGGUUUAGGGaGUGUACCUCAG 3' } \\ \text { hsa-let-7b } & \text { 3' UUGGUGUGUUGGAUGAUGGAGU 5' }\end{aligned}$

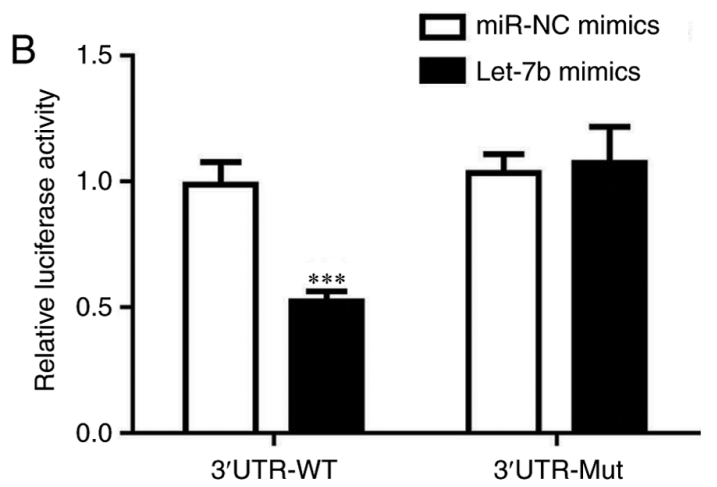

Figure 5. CDK6 is a direct target of let-7b. (A) Sequence alignment of let-7b to the 3'UTR of CDK6 mRNA. (B) Transfection of let-7b mimics decreased the relative luciferase activity of cells transfected with CDK6 3'UTR-WT, but not 3'UTR-Mut. ${ }^{* * *} \mathrm{P}<0.0001$. CDK6, cyclin-dependent kinase 6; 3'UTR, 3' untranslated region; WT, wild-type; Mut, mutant; miR, microRNA; NC, negative control.

HOST2 regulates CDK6 via the repression of let- $7 b$. lncRNAs can function as sponge RNAs to regulate gene expression (17). A previous study revealed that HOST2 repressed the expression of let-7b in breast cancer (18). To determine whether HOST 2 controlled CDK6 levels through the sponging of let-7b, the expression of HOST2 was silenced in the TNBC cells. The knockdown of HOST2 increased the expression levels of let-7b in the MDA-MB-231 and MDA-MB-468 cells (Fig. 4A). To further validate the regulation of let-7b by HOST2, another independent HOST2 siRNA (HOST2 siRNA\#) was used to knock down HOST2 in the TNBC cells. Consistent with the HOST siRNA, transfection with HOST2 siRNA\# also increased the expression of let-7b (Fig. 4B and C). In addition, the transfection of let-7b mimics reduced the luciferase activity of MDA-MB-231 cells transfected with pGL3-HOST2 WT containing a putative binding site for let-7b (Fig. 4D). Similarly, in the MDA-MB-468 cells, the overexpression of let-7b repressed luciferase activity in the cells transfected with pGL3-HOST2 WT (Fig. 4E), indicating that HOST2 directly repressed the expression of let-7b. In addition, the enhanced expression of let-7b caused by transfection of let-7b mimics (Fig. 4F) reduced the expression of CDK6 at the mRNA and protein levels in the MDA-MB-231 and MDA-MB-468 cells (Fig. 4G and $\mathrm{H}$ ). These findings suggested that HOST2 controlled the expression of CDK6 through sponging of let-7b in the TNBC cells.

Let-7b directly binds to the 3'UTR of CDK6 $\mathrm{mRNA}$. Whether let-7b directly regulated the level of CDK6 was investigated. The prediction of let-7b binding sites indicated that let-7b may directly bind to the 3'UTR of CDK6 mRNA (Fig. 5A). A dual luciferase assay was used, which demonstrated that the overexpression of let-7b decreased the relative luciferase activity of cells transfected with CDK6 3'UTR-WT, but not 3'UTR-Mut (Fig. 5B). These results confirmed that let-7b targeted the 3'UTR of CDK6 mRNA.

Expression of let-7b is negatively associated with expression levels of HOST2 and CDK6 in TNBC tumor tissues. To further evaluate the clinical significance of HOST2/let7b/CDK6 in TNBC, RT-qPCR analysis was performed to detect the expression of let-7b, and Pearson's correlation analysis was used to analyze the results. Compared with matched normal tissues, decreased expression of let-7b was observed in TNBC tumor tissues (Fig. 6A). Additionally, a significant negative correlation was identified between the expression of let-7b and HOST2, and the expression of let-7b and CDK6 in TNBC tumor tissues (Fig. 6B and C).

\section{Discussion}

TNBC is characterized by an aggressive phenotype and a high rate of relapse (19). In the absence of well-defined molecular targets, chemotherapy is the first line of treatment for TNBC, but this often results in chemoresistance, and the mortality rate remains high (20). Several reports have indicated that lncRNAs are important regulators of TNBC progression (21-23). In the present study, lncRNA-HOST2 was identified as an oncogene in TNBC, and may provide a novel therapeutic target for TNBC.

HOST2 was first defined in human ovarian cancer and functions as an oncogene through binding to let-7b $(24,25)$. HOST 2 has been demonstrated to regulate cell proliferation, migration, invasion and cell apoptosis in human osteosarcoma and hepatocellular carcinoma cells $(12,26)$. A previous study showed that HOST2 was overexpressed in breast cancer and that the silencing of HOST2 inhibited the migration and invasion of MCF7 cells, a luminal breast cancer cell line (18). Through comparison of the expression of HOST in TNBC tumor tissues and matched normal tissues, a marked increase in HOST2 was revealed in TNBC tumor tissues. In addition, the expression of HOST2 was significantly increased in TNBC cell lines compared with normal breast epithelial cells. The silencing of HOST2 decreased the rate of cell proliferation of the two TNBC cell lines. Flow cytometric analysis indicated that HOST2 silencing led to an enrichment of cells in the $G_{0} / G_{1}$ phase, but did not significantly elevate apoptotic cell rate, suggesting that HOST2 may promote TNBC proliferation via accelerating the cell cycle.

The above results of the cell cycle assay showed that HOST2 regulated the $\mathrm{G}_{1} / S$ phase in cells. Through a literature review, it was noted that CDK6 is a major $\mathrm{G}_{0} / \mathrm{G}_{1}$ checkpoint regulator and frequently deregulated in cancer cells (27); the expression of CDK6 has been associated with poor survival outcomes and is considered to be a promising therapeutic 

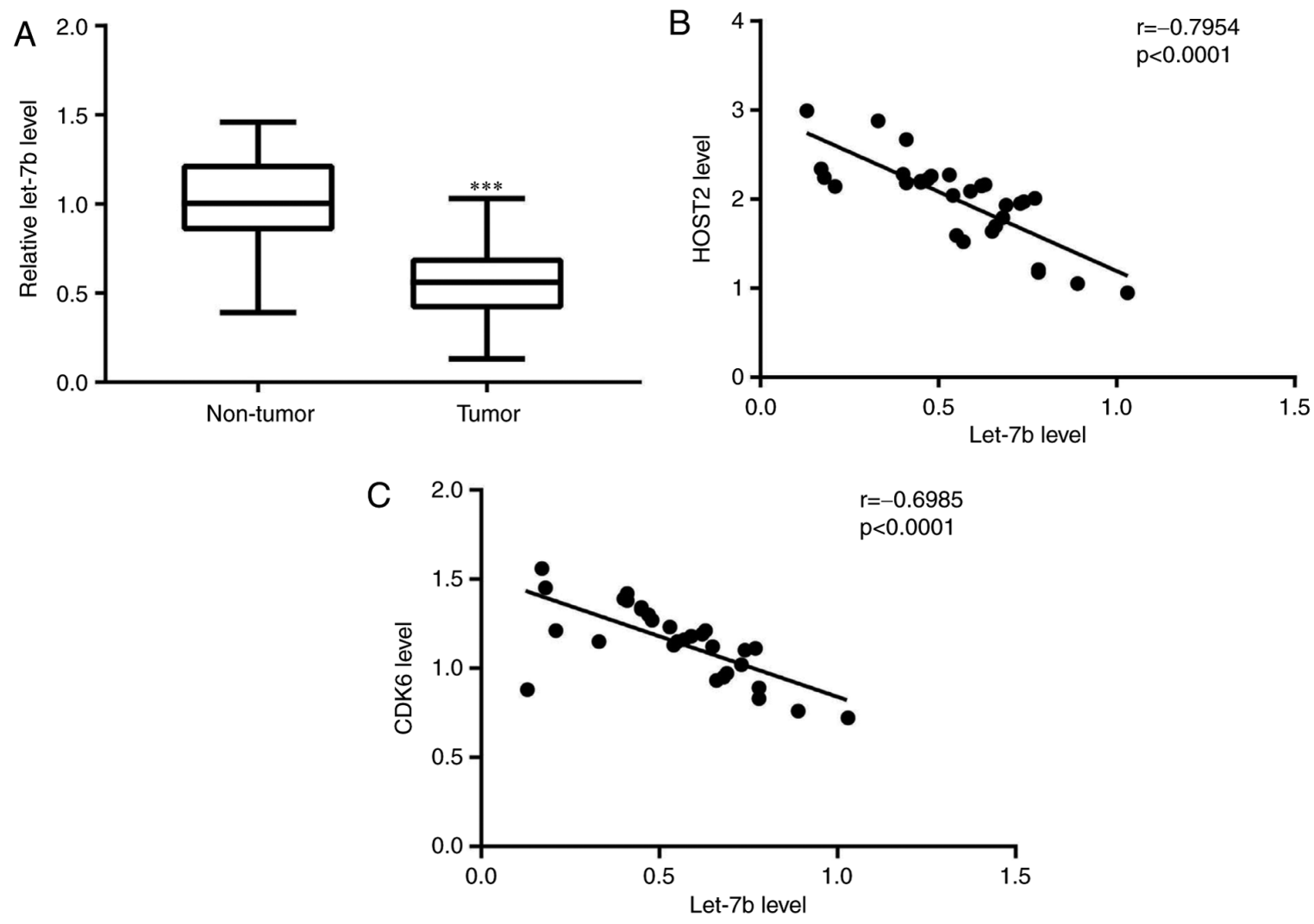

Figure 6. Expression of let-7b is inversely correlated with HOST2 and CDK6 in tumor tissues from patients with TNBC. (A) Compared with matched normal tissues, let-7b levels were lower in tumor tissues from patients with TNBC. (B) Pearson's analysis of let-7b levels with HOST2 levels showed an inverse correlation. (C) Pearson's analysis of let-7b levels with CDK6 levels showed a negative correlation. ${ }^{* * *} \mathrm{P}<0.0001$. TNBC, triple negative breast cancer; HOST, human ovarian cancer-specific transcript 2; CDK, cyclin-dependent kinase.

target for TNBC (28). The results suggested that CDK6 was a key regulator in the $\mathrm{G}_{1} / \mathrm{S}$ phase and is pivotal in the progression of TNBC. Other IncRNAs, including gadd7, GAS5 and MYU, have been reported to regulate the expression of CDK6 in different cellular contexts (29-31). In the present study, following the silencing of HOST2, the expression of $\mathrm{G}_{1} / \mathrm{S}$ cell cycle regulator CDK6, was detected. It was found that CDK6 was downregulated following HOST silencing, and a positive correlation between the expression of HOST2 and CDK6 was identified in the TNBC tumour tissues. In addition, the silencing of HOST2 decreased the expression of CDK6 in TNBC cell lines, indicating that HOST2 may enhance the expression of CDK6. The regulatory association between HOST2 and CDK6 may explain the cell cycle redistribution following HOST2 knockdown. A previous study showed that several lncRNAs function as potent miRNA sponges to regulate gene expression (32). It has been suggested that abundant lncRNAs can sequester miRNAs away from their targeted mRNAs (33). A previous study showed that let-7b was a target of HOST2 (25). The present study further confirmed that HOST2 sponged let-7b to downregulate its expression in MDA-MB-231 and MDA-MB-468. In addition, bioinformatics analysis indicated complementary sequences between let-7b and the 3'UTR of CDK6 mRNA. A dual luciferase assay confirmed let- $7 \mathrm{~b}$ as a direct regulator of $\mathrm{CDK} 6$, and the results of western blot and RT-qPCR analyses confirmed that let-7b suppressed the expression of CDK6. Of note, the expression of let-7b was downregulated in TNBC tumour tissues, and its expression was negatively correlated with that of CDK6 and HOST2.

In conclusion, the present study suggested that an HOST2/let-7b/CDK6 expression axis is involved in the promotion TNBC proliferation. Mechanistically, downregulation of the expression of HOST2 resulted in increased expression of let-7b, a tumor suppressor, and decreased expression of CDK6, resulting in the alteration of cell cycle distribution. Therefore, HOST2 may be a candidate therapeutic target for the treatment of patients with TNBC in the near future.

\section{Acknowledgements}

Not applicable.

\section{Funding}

No funding was received.

\section{Availability of data and materials}

Details of data and materials are available from the correspondence author on request.

\section{Authors' contributions}

YucZ was responsible for study design. YueZ, HZ and YuZ performed most of experiments. HK, WH helped with collection 
of clinical samples. YucZ supervised the study and prepared the manuscript. All authors read and approved the final manuscript.

\section{Ethics approval and consent to participate}

The present study was approved by the Ethics Committee of Wuhai People's Hospital. Informed consent regarding the use of tissue samples was obtained from each patient.

\section{Patient consent for publication}

Not applicable.

\section{Competing interests}

The authors confirm that they have no competing interests.

\section{References}

1. Schettini F, Buono G, Cardalesi C, Desideri I, De Placido S and Del Mastro L: Hormone receptor/human epidermal growth factor receptor 2-positive breast cancer: Where we are now and where we are going. Cancer Treat Rev 46: 20-26, 2016.

2. Zheng S, Bai JQ, Li J, Fan JH, Pang Y, Song QK, Huang R, Yang HJ, Xu F, Lu N and Qiao YL: The pathologic characteristics of breast cancer in China and its shift during 1999-2008: a national-wide multicenter cross-sectional image over 10 years. Int J Cancer 131: 2622-2631, 2012

3. Fan L, Strasser-Weippl K, Li JJ, St Louis J, Finkelstein DM, Yu KD, Chen WQ, Shao ZM and Goss P: Breast cancer in China. Lancet Oncol 15: e279-e289, 2014.

4. Comprehensive molecular portraits of human breast tumours. Nature 490: 61-70, 2012.

5. Agrawal LS and Mayer IA: Platinum agents in the treatment of early-stage triple-negative breast cancer: Is it time to change practice? Clin Adv Hematol Oncol 12: 654-658, 2014.

6. Foulkes WD, Smith IE and Reis-Filho JS: Triple-negative breast cancer. N Engl J Med 363: 1938-1948, 2010.

7. Pinto R, De Summa S, Pilato B and Tommasi S: DNA methylation and miRNAs regulation in hereditary breast cancer: Epigenetic changes, players in transcriptional and post-transcriptional regulation in hereditary breast cancer. Curr Mol Med 14: 45-57, 2014.

8. Maruyama R and Suzuki H: Long noncoding RNA involvement in cancer. BMB Rep 45: 604-611, 2012.

9. Li JP, Liu LH, Li J, Chen Y, Jiang XW, Ouyang YR, Liu YQ, Zhong H, Li H and Xiao T: Microarray expression profile of long noncoding RNAs in human osteosarcoma. Biochem Biophys Res Commun 433: 200-206, 2013.

10. Lv M, Xu P, Wu Y, Huang L, Li W, Lv S, Wu X, Zeng X, Shen R, Jia X, et al: LncRNAs as new biomarkers to differentiate triple negative breast cancer from non-triple negative breast cancer. Oncotarget 7: 13047-13059, 2016.

11. Rinn JL and Chang HY: Genome regulation by long noncoding RNAs. Annu Rev Biochem 81: 145-166, 2012.

12. Liu RT, Cao JL, Yan CQ, Wang Y, An CJ and Lv HT: Effects of LncRNA-HOST2 on cell proliferation, migration, invasion and apoptosis of human hepatocellular carcinoma cell line SMMC-7721. Biosci Rep 37, 2017.

13. Zhong Y, Gao D, He S, Shuai C and Peng S: Dysregulated expression of long noncoding RNAs in ovarian cancer. Int J Gynecol Cancer 26: 1564-1570, 2016.
14. Livak KJ and Schmittgen TD: Analysis of relative gene expression data using real-time quantitative PCR and the $2-\Delta \Delta C T$ method. Methods 25: 402-408, 2001.

15. Malumbres $\mathbf{M}$ and Barbacid M: Mammalian cyclin-dependent kinases. Trends Biochem Sci 30: 630-641, 2005.

16. Lim S and Kaldis P: Cdks, cyclins and CKIs: roles beyond cell cycle regulation. Development 140: 3079-3093, 2013.

17. Qi X, Zhang DH, Wu N, Xiao JH, Wang X and Ma W: ceRNA in cancer: Possible functions and clinical implications. J Med Genet 52: 710-718, 2015.

18. Lu PW, Li L, Wang F and Gu YT: Effects of long non-coding RNA HOST2 on cell migration and invasion by regulating microRNA let-7b in breast cancer. J Cell Biochem 119: 4570-4580, 2017.

19. Palma G, Frasci G, Chirico A, Esposito E, Siani C, Saturnino C, Arra C, Ciliberto G, Giordano A and D'Aiuto M: Triple negative breast cancer: Looking for the missing link between biology and treatments. Oncotarget 6: 26560-26574, 2015.

20. Oakman C, Viale G and Di Leo A: Management of triple negative breast cancer. Breast 19: 312-321, 2010.

21. Zhang Y, He Q, Hu Z, Feng Y, Fan L, Tang Z, Yuan J, Shan W, Li C, Hu X, et al: Long noncoding RNA LINP1 regulates repair of DNA double-strand breaks in triple-negative breast cancer. Nat Struct Mol Biol 23: 522-530, 2016.

22. Lin A, Li C, Xing Z, Hu Q, Liang K, Han L, Wang C, Hawke DH, Wang S, Zhang Y, et al: The LINK-A lncRNA activates normoxic HIFlalpha signalling in triple-negative breast cancer. Nat Cell Biol 18: 213-224, 2016.

23. Pickard MR and Williams GT: Regulation of apoptosis by long non-coding RNA GAS5 in breast cancer cells: implications for chemotherapy. Breast Cancer Res Treat 145: 359-370, 2014.

24. Rangel LB, Sherman-Baust CA, Wernyj RP, Schwartz DR, Cho KR and Morin PJ: Characterization of novel human ovarian cancer-specific transcripts (HOSTs) identified by serial analysis of gene expression. Oncogene 22: 7225-7232, 2003.

25. Gao Y, Meng H, Liu S, Hu J, Zhang Y, Jiao T, Liu Y, Ou J, Wang D, Yao L, et al: LncRNA-HOST2 regulates cell biological behaviors in epithelial ovarian cancer through a mechanism involving microRNA let-7b. Hum Mol Genet 24: 841-852, 2015.

26. Wang W, Li X, Meng FB, Wang ZX, Zhao RT and Yang CY: Effects of the long non-coding RNA HOST2 on the proliferation, migration, invasion and apoptosis of human osteosarcoma cells. Cell Physiol Biochem 43: 320-330, 2017.

27. Tadesse S, Yu M, Kumarasiri M, Le BT and Wang S: Targeting CDK6 in cancer: State of the art and new insights. Cell Cycle 14: 3220-3230, 2015.

28. Hsu YH, Yao J, Chan LC, Wu TJ, Hsu JL, Fang YF, Wei Y, Wu Y, Huang WC, Liu CL, et al: Definition of PKC-alpha, CDK6, and MET as therapeutic targets in triple-negative breast cancer. Cancer Res 74: 4822-4835, 2014.

29. Liu X, Li D, Zhang W, Guo M and Zhan Q: Long non-coding RNA gadd7 interacts with TDP-43 and regulates Cdk6 mRNA decay. EMBO J 31: 4415-4427, 2012.

30. Liu Z, Wang W, Jiang J, Bao E, Xu D, Zeng Y, Tao L and Qiu J: Downregulation of GAS5 promotes bladder cancer cell proliferation, partly by regulating CDK6. PLoS One 8: e73991, 2013.

31. Kawasaki Y, Komiya M, Matsumura K, Negishi L, Suda S, Okuno M, Yokota N, Osada T, Nagashima T, Hiyoshi M, et al: MYU, a target lncRNA for Wnt/c-Myc signaling, mediates induction of CDK6 to promote cell cycle progression. Cell Rep 16: 2554-2564, 2016.

32. 32. Hansen TB, Jensen TI, Clausen BH, Bramsen JB, Finsen B, Damgaard CK and Kjems J: Natural RNA circles function as efficient microRNA sponges. Nature 495: 384-388, 2013.

33. Salmena L, Poliseno L, Tay Y, Kats L and Pandolfi PP: A ceRNA hypothesis: the Rosetta Stone of a hidden RNA language? Cell 146: 353-358, 2011. 